\title{
Application of Low Temperature during the Malaxation Phase of Virgin Olive Oil Mechanical Extraction Processes of Three Different Italian Cultivars
}

\author{
Gianluca Veneziani ${ }^{1}$, Davide Nucciarelli ${ }^{1}$, Agnese Taticchi ${ }^{1}$, Sonia Esposto ${ }^{1, *}$, Roberto Selvaggini ${ }^{1}$, \\ Roberto Tomasone $^{2}$, Mauro Pagano ${ }^{2}$ and Maurizio Servili ${ }^{1}$
}

1 Department of Agricultural, Food and Environmental Sciences, University of Perugia, Via S. Costanzo, 06126 Perugia, Italy; gianluca.veneziani@unipg.it (G.V.); davide.nucciarelli@unipg.it (D.N.); agnese.taticchi@unipg.it (A.T.); roberto.selvaggini@unipg.it (R.S.); maurizio.servili@unipg.it (M.S.)

2 Council for Agricultural Research and Economics Research, Centre for Engineering and Agro-Food Processing, 00015 Monterotondo, Italy; roberto.tomasone@crea.gov.it (R.T.); mauro.pagano@crea.gov.it (M.P.)

* Correspondence: sonia.esposto@unipg.it

Citation: Veneziani, G.; Nucciarelli, D.; Taticchi, A.; Esposto, S.; Selvaggini, R.; Tomasone, R.; Pagano, M.; Servili, M. Application of Low Temperature during the Malaxation Phase of Virgin Olive Oil Mechanical Extraction Processes of Three Different Italian Cultivars. Foods 2021, 10, 1578. https://doi.org/10.3390/ foods 10071578

Academic Editor:

Verica Dragović-Uzelac

Received: 7 June 2021

Accepted: 5 July 2021

Published: 7 July 2021

Publisher's Note: MDPI stays neutral with regard to jurisdictional claims in published maps and institutional affiliations.

Copyright: (c) 2021 by the authors. Licensee MDPI, Basel, Switzerland. This article is an open access article distributed under the terms and conditions of the Creative Commons Attribution (CC BY) license (https:/ / creativecommons.org/licenses/by/ $4.0 /)$.

\begin{abstract}
The malaxation step, one of the most important phases of the virgin olive oil (VOO) mechanical extraction process involved in the development of the main quality characteristics of the final product, was carried out at a low temperature $\left(18^{\circ} \mathrm{C}\right)$. The rapid control of malaxer temperature was handled with the same chiller as that of the heat exchanger used in a semi-industrial extraction plant. Low temperature was used during the full olive paste kneading process and also for half of this process, which showed that there was a significant impact on the phenolic and volatile contents of VOO. Trials were conducted on three different cultivars (Canino, Moraiolo and Peranzana), and their phenolic and volatile concentrations showed different quantitative and qualitative effects due to the prolonged use of low temperature after the crushing phase, as a function of the different genetic origins of the olives. The process of phenolic compound solubilization into the oily phase was negatively influenced by the use of low temperature during the entire malaxation period for all the cultivars, whereas the volatile fraction showed an improvement in VOO flavor mainly due to the oil extracted from Canino olives.
\end{abstract}

Keywords: low temperature; malaxation; VOO quality; hydrophilic phenols; volatile compounds

\section{Introduction}

In recent years, the thermal conditioning of olive paste during the olive oil extraction process has increased in usage due to the different impact in oil yield and the quality characteristics of the final product. Previous studies focused their attention on the biochemical process, showing that a differentiated activity level of endogenous enzymes occurred due to the application of different processing temperatures [1-6]. The possibility of accurate thermal control and management of temperature conditions during crushing and malaxation phases stimulated the investigation of different cultivar behaviors during the virgin olive oil (VOO) extraction process [7,8], to discover the best combinations of process parameters that influence the chemical characteristics and sensorial properties affecting VOO quality. The optimization of technological variables plays an important role in the modulation of physical, chemical and biochemical processes with the aim of improving VOO qualitative standards. One of the most important parameters of the VOO mechanical extraction process is the processing temperature. This parameter can modify and regulate the different enzyme activities due to the endogenous enzymes present in the constitutive parts of olive drupe, such as depolymerizing enzymes (pectinase, cellulase and hemicellulase), poly-phenol-oxydase (PPO), peroxidase (POD) and lipoxygenase (LOX). The possibility of controlling the temperature during the entire extraction process allows the opportunity to regulate the biochemical reactions of some endogenous enzymes that have 
a high impact on VOO quality, which is related to the health and sensory characteristics of the final product $[4,5]$.

The application of high temperature favors the degradative process of phenolic compounds induced by PPO and POD and promotes enzymatic activities, which increases the oxidation process during the mechanical extraction of VOO. The high temperature of fruits before the extraction process, the increasing temperature of olive paste during crushing and, most of all, the regulation of malaxation temperature are all factors that can strictly influence the activity level of PPO and POD. For this reason, many studies have been performed to optimize the processing temperature in order to partially control the final phenolic content of the product during the different steps of the extraction process $[3,4,9]$.

On the other hand, the most important fraction of volatile compounds that guarantees the development of VOO flavor is obtained in the first phases of the technological process, during crushing and immediately thereafter. A minor percentage is also produced during the kneading phase of olive paste, as reported in previous studies $[10,11]$. The lipoxygenase pathway extracted from olive fruit showed a high activity level at temperatures ranging from $15{ }^{\circ} \mathrm{C}$ to $40{ }^{\circ} \mathrm{C}[12,13]$, but the best performances were obtained at temperatures $\leq 20^{\circ} \mathrm{C}$ [13-15]. These results were mainly due to the optimum temperature of hydroperoxide lyase (HPL), which was detected between $15^{\circ} \mathrm{C}$ and $20^{\circ} \mathrm{C}$ and showed a high increase in $C_{5}$ and $C_{6}$ compounds [13]. As demonstrated by different studies, employing the thermal treatment of olives and/or crushed olive pastes with a low temperature increased the concentration of volatile compounds, improving the fruity and herbaceous sensory notes of VOO extracted from different olive cultivars $[14,16,17]$. However, the impact was highly differentiated, strictly related to the genetic origin of the fruits and influenced by the specific lipoxygenase activity load of the cultivar [8-11]. The quantitative and qualitative variability of the volatile fractions of saturated and unsaturated $C_{5}$ and $C_{6}$ aldehydes, alcohols and esters was influenced not only by the cultivars but also by other factors, such as the ripening stages of the fruit, agricultural practices, growing areas, and climatic and technological aspects $[18,19]$.

Currently, the best way to manage the temperature of extraction plants at an industrial scale is to employ the rapid conditioning of olive paste after the crushing step with a heat exchanger $[8,17,20-22]$. The main industrial manufacturers of VOO extraction systems produce different models of heat exchanger, which are fully used by operators in the VOO sector.

Probably due to the different temperature values, this group of endogenous enzymes works differently, and an interesting research topic is to examine how the specific activity that characterizes each different cultivar is influenced by the olive oil paste exposure time at low temperature. The research design was carried out on three different cultivars characterized by different concentrations of phenolic and volatile compounds, from a quantitative and qualitative point of view, in order to better evaluate the impact of innovative technology on VOOs obtained by olive fruits of different genetic origin. The main aim of the work concerned the use of temperatures below $20^{\circ} \mathrm{C}$ during the malaxation phase to assess the potential effect on the development of volatile compounds and on the phenol content of VOO in relation to the different genetic origins of the raw material.

\section{Materials and Methods}

\subsection{Chemicals}

The seco-iridoid derivatives and lignans were extracted from VOO, as described by Selvaggini et al. (2014). The chemical standards, solvents and other compounds used during the analyses of VOO were supplied by Merck (Merck KGaA, Darmstadt, Germany), tyrosol was supplied by Cabru s.a.s. (Arcore, Milan, Italy) and hydroxy-tyrosol was supplied by Fluka (Milan, Italy). 


\subsection{VOO Mechanical Extraction Process}

The olives belonged to three different Italian cultivars (Canino, Moraiolo and Peranzana) and were processed within $48 \mathrm{~h}$ after the harvesting period and from the middle of October to the first week of November 2019. The olive growing areas of Canino and Peranzana were located in the Lazio and Apulia regions, respectively, whereas the olives of Moraiolo were harvested in the Umbria region. VOOs were extracted from approximately $180 \mathrm{~kg}$ of olives using an industrial plant TEM 200 system (Toscana Enologica Mori, Tavarnelle Val di Pesa, Florence, Italy) equipped with a hammer mill, a malaxer with a gas controller system that had a working capacity of $200 \mathrm{~kg}$ of olives, and a two-phase decanter. The final separation step was carried out with a UVPX 305 AGT 14 centrifuge (Alfa Laval S.p.A., Tavarnelle Val di Pesa, Florence, Italy). Rapid control of olive paste after the crushing phase was obtained using an EVO-Line heat exchanger (Alfa Laval S.p.A.). The same chiller system as the heat exchanger was also used to regulate the temperature of the malaxer during the $30 \mathrm{~min}$ kneading phase. The control trials (C-VOO) were conducted at $25^{\circ} \mathrm{C}$, whereas the experimental tests were conducted by settling the temperature of the heat exchanger at $18{ }^{\circ} \mathrm{C}$ and changing the temperature of the malaxer as follows:

- Control (C-VOO) heat exchanger at $25^{\circ} \mathrm{C}$, malaxer at $25^{\circ} \mathrm{C}$ for $30 \mathrm{~min}$;

- Test 1 (T1-VOO) heat exchanger at $18^{\circ} \mathrm{C}$, malaxer at $25^{\circ} \mathrm{C}$ for $30 \mathrm{~min}$;

- $\quad$ Test 2 (T2-VOO) heat exchanger at $18{ }^{\circ} \mathrm{C}$, malaxer at $18^{\circ} \mathrm{C}$ for $30 \mathrm{~min}$;

- Test 3 (T3-VOO) heat exchanger at $18{ }^{\circ} \mathrm{C}$, malaxer at $18^{\circ} \mathrm{C}$ for $15 \mathrm{~min}+15 \mathrm{~min}$ at $25^{\circ} \mathrm{C}$.

\subsection{VOO Chemical Analysis}

\subsubsection{Legal Quality Parameters}

The main legal quality parameter methods (free fatty acids, peroxide value, K232, $\mathrm{K} 270$ and $\Delta \mathrm{K}$ ) were evaluated according to Regulation (EU) 2015/1830 [23].

\subsubsection{Phenolic Compounds}

The content of the main hydrophilic phenols of VOO controls and extracts at low temperature was analyzed with high-performance liquid chromatography (HPLC) using the same instrument and the same method as described by Taticchi et al. (2021). The concentrations of 3,4-DHPEA-EDA (oleacein), p-HPEA-EDA (oleocanthal), 3,4-DHPEAEA (isomer of the oleuropein aglycon), p-HPEA-EA (ligstroside aglycon), 3,4-DHPEA (hydroxy-tyrosol), p-HPEA (tyrosol), vanillic acid, p-cumaric acid, (+)-1-acetoxypinoresinol and (+)-pino-resinol were expressed in $\mathrm{mg} / \mathrm{kg}$ of oil.

\subsubsection{Volatile Compounds}

The volatile fractions of the VOO control and extracts at low temperature were analyzed with headspace solid-phase microextraction followed by gas chromatography-mass spectrometry (HS-SPME/GC-MS) using an Agilent Technologies GC 7890B instrument equipped with a "Multimode Injector" (MMI) 7693A (Agilent Technologies, Santa Clara, CA, USA) and followed the method described by Taticchi et al. [24]. The concentrations of aldehydes (pentanal, (E)-2-pentenal, hexanal, (E)-2-hexenal, (E,E)-2,4-hexadienal), alcohols (1-pentanol, 1-penten-3-ol, (E)-2-penten-1-ol, (Z)-2-penten-1-ol, 1-hexanol, (E)-2-hexen-1-ol, (Z)-3-hexen-1- ol), esters (hexyl acetate (Z)-3-hexenyl acetate) and ketones (3-pentanone, 1-penten-3-one and 6-methyl-5-hepten-2-one) were expressed in $\mu \mathrm{g} / \mathrm{kg}$ of oil.

\subsection{Statistical Analysis}

All the phenolic and volatile compound data of the VOO controls and experiments were analyzed by SigmaPlot Software 12.3 (Systat Software Inc., San Jose, CA, USA) with one-way analysis of variance (ANOVA). 


\section{Results and Discussion}

Based on the results, in the mechanical extraction process of VOO, the use of low temperature during the first phases, such as the crushing and malaxation of olive paste phases, showed that the temperature had a significant role in controlling the activities of the main endogenous enzymes (LOX, PPO and POD) that regulate both the neoformation of volatile compounds and the oxidation phenomenon of the phenolic fraction $[3,5,8,16,17]$. For these reasons, the research design compared three different cultivars to better assess the effects of the low temperature in relation to different olive fruits characterized by different levels of enzymatic activity and different concentrations of phenolic and volatile compounds. Currently, the use of a heat exchanger to decrease the temperature after the crushing step is widespread, and its chiller system has also been utilized to reduce the temperature of the malaxer, which extended the treatment at low temperature during the kneading of olive paste in order to evaluate its impact on VOO quality.

The use of low temperature during the malaxation phase did not modify the oil yield, which confirmed the data reported in a previous study that did not observe obstacles to the coalescence phenomena of oil droplets and VOO extractability [17].

Compared with the control test results, the assessment of the quality indices of T1, T2 and T3-VOOs did not show significant differences for all three cultivars, with values of free fatty acids, peroxide, $K_{232}, K_{270}$ and $\Delta K$ in line with the legal limit [23] (Table 1).

Table 1. Legal quality parameters of VOOs extracted at different processing temperatures.

\begin{tabular}{|c|c|c|c|c|}
\hline & C-VOO & T1-VOO & T2-VOO & T3-VOO \\
\hline & Canino & & & \\
\hline Free fatty acid ( $\%$ oleic acid $)^{1}$ & $0.29 \pm 0.01 a$ & $0.28 \pm 0.01 a$ & $0.26 \pm 0.01 \mathrm{a}$ & $0.26 \pm 0.01 a$ \\
\hline Peroxide value $\left(\mathrm{meqO}_{2} / \mathrm{kg}\right)$ & $8.9 \pm 2.8 \mathrm{a}$ & $6.8 \pm 1.1 \mathrm{a}$ & $8.4 \pm 0.3 \mathrm{a}$ & $6.7 \pm 0.1 \mathrm{a}$ \\
\hline $\mathrm{K}_{232}$ & $1.78 \pm 0.32 \mathrm{a}$ & $1.63 \pm 0.04 \mathrm{a}$ & $1.71 \pm 0.03 \mathrm{a}$ & $1.64 \pm 0.08 \mathrm{a}$ \\
\hline$K_{270}$ & $0.11 \pm 0.004 \mathrm{a}$ & $0.13 \pm 0.01 \mathrm{a}$ & $0.12 \pm 0.01 \mathrm{a}$ & $0.14 \pm 0.003 a$ \\
\hline \multirow[t]{2}{*}{$\Delta \mathrm{K}$} & $-0.002 \pm 0.0004 a$ & $-0.002 \pm 0.0002 \mathrm{a}$ & $-0.002 \pm 0.0004 \mathrm{a}$ & $-0.003 \pm 0.0004 a$ \\
\hline & Moraiolo & & & \\
\hline Free fatty acid (\% oleic acid) ${ }^{1}$ & $0.29 \pm 0.01 \mathrm{a}$ & $0.29 \pm 0.01 a$ & $0.32 \pm 0.02 \mathrm{a}$ & $0.3 \pm 0.01 \mathrm{a}$ \\
\hline Peroxide value $\left(\mathrm{meqO}_{2} / \mathrm{kg}\right)$ & $6.1 \pm 1.1 \mathrm{a}$ & $5.5 \pm 0.3 \mathrm{a}$ & $7.3 \pm 1.3 \mathrm{a}$ & $5.8 \pm 0.5 a$ \\
\hline $\mathrm{K}_{232}$ & $1.81 \pm 0.08 \mathrm{a}$ & $1.72 \pm 0.04 a$ & $1.72 \pm 0.1 \mathrm{a}$ & $1.68 \pm 0.02 a$ \\
\hline $\mathrm{K}_{270}$ & $0.12 \pm 0.01 \mathrm{a}$ & $0.12 \pm 0.01 \mathrm{a}$ & $0.12 \pm 0.01 \mathrm{a}$ & $0.11 \pm 0 \mathrm{a}$ \\
\hline \multirow[t]{2}{*}{$\Delta \mathrm{K}$} & $-0.003 \pm 0.0004 a$ & $-0.003 \pm 0.0004 a$ & $-0.003 \pm 0.0004 \mathrm{a}$ & $-0.003 \pm 0.0004 \mathrm{a}$ \\
\hline & Peranzana & & & \\
\hline Free fatty acid (\% oleic acid) ${ }^{1}$ & $0.28 \pm 0.01 a$ & $0.28 \pm 0.01 \mathrm{a}$ & $0.3 \pm 0.01 \mathrm{a}$ & $0.3 \pm 0.01 \mathrm{a}$ \\
\hline Peroxide value $\left(\mathrm{meqO}_{2} / \mathrm{kg}\right)$ & $8.1 \pm 2.3 a$ & $8.2 \pm 2.6 a$ & $8.9 \pm 0.7 \mathrm{a}$ & $6.4 \pm 0.1 \mathrm{a}$ \\
\hline $\mathrm{K}_{232}$ & $2.07 \pm 0.21 \mathrm{a}$ & $1.9 \pm 0.16 a$ & $1.93 \pm 0.08 \mathrm{a}$ & $1.71 \pm 0.02 \mathrm{a}$ \\
\hline$K_{270}$ & $0.16 \pm 0.002 \mathrm{a}$ & $0.14 \pm 0.01 \mathrm{a}$ & $0.15 \pm 0.001 \mathrm{a}$ & $0.12 \pm 0.002 \mathrm{a}$ \\
\hline$\Delta \mathrm{K}$ & $-0.002 \pm 0.0002 \mathrm{a}$ & $-0.002 \pm 0.003 a$ & $-0.003 \pm 0.0002 \mathrm{a}$ & $-0.002 \pm 0.0004 \mathrm{a}$ \\
\hline
\end{tabular}

${ }^{1}$ The data are the mean values of two independent extractions, \pm standard deviation. The values in each row having different letters (a) are significantly different from one another $(p<0.05) . \mathrm{C}=$ control (heat exchanger at $25^{\circ} \mathrm{C}$, malaxer at $25^{\circ} \mathrm{C}$ for $30 \mathrm{~min}$ ); $\mathrm{T} 1=$ test 1 (heat exchanger at $18{ }^{\circ} \mathrm{C}$, malaxer at $25^{\circ} \mathrm{C}$ for $30 \mathrm{~min}$ ); $\mathrm{T} 2=$ test 2 (heat exchanger at $18^{\circ} \mathrm{C}$, malaxer at $18{ }^{\circ} \mathrm{C}$ for 30 min); $\mathrm{T} 3=$ test 3 (heat exchanger at $18{ }^{\circ} \mathrm{C}$, malaxer at $18^{\circ} \mathrm{C}$ for $15 \mathrm{~min}+15 \mathrm{~min}$ at $25^{\circ} \mathrm{C}$ ).

\subsection{Test 1-Use of Low Temperature after Crushing Phase}

The use of flash thermal conditioning of olive paste after the crushing phase (T1-VOO), induced by a heat exchanger, allowed for the positive variation in the phenolic fraction to be determined, which was a function of the genetic origin of the olives. The settling of the heat exchanger at $18{ }^{\circ} \mathrm{C}$ confirmed data that indicated a significant improvement in the phenolic composition for the T1-VOOs extracted from the three cultivars in comparison with the C-VOOs [8]. The phenolic content recorded increases of $44.3 \%, 82 \%$ and $14.8 \%$ for the Canino, Moraiolo and Peranzana cultivars, respectively (Table 2). 
Table 2. Phenolic composition $(\mathrm{mg} / \mathrm{kg})$ of VOOs extracted at different processing temperatures.

\begin{tabular}{|c|c|c|c|c|}
\hline & C-VOO & T1-VOO & T2-VOO & T3-VOO \\
\hline $\begin{array}{l}\text { 3,4-DHPEA }{ }^{1} \\
p \text {-HPEA } \\
\text { Vanilic acid } \\
p \text {-Cumaric acid } \\
\text { 3,4-DHPEA-EDA } \\
p \text {-HPEA-EDA } \\
\text { (+)-1-acetoxypinoresinol } \\
\text { (+)-pinoresinol } \\
\text { 3,4-DHPEA-EA } \\
\text { Ligstroside aglycone } \\
\text { Total phenols }\end{array}$ & $\begin{array}{l}\text { Canino } \\
1.2 \pm 0.1 \mathrm{a} \\
1.1 \pm 0.2 \mathrm{a} \\
0.1 \pm 0 \mathrm{a} \\
\text { n.d. } \\
87.6 \pm 5.4 \mathrm{a} \\
20 \pm 0.6 \mathrm{a} \\
9.8 \pm 1.1 \mathrm{a} \\
10.4 \pm 1.1 \mathrm{a} \\
9.4 \pm 0.8 \mathrm{a} \\
6 \pm 0.3 \mathrm{a} \\
145.6 \pm 5.8 \mathrm{a}\end{array}$ & $\begin{array}{l}1.1 \pm 0.1 \mathrm{a} \\
1.6 \pm 0.5 \mathrm{ab} \\
0.2 \pm 0.1 \mathrm{a} \\
\text { n.d. } \\
129.2 \pm 14.1 \mathrm{~b} \\
31.4 \pm 2.4 \mathrm{~b} \\
11.1 \pm 0.8 \mathrm{a} \\
11.3 \pm 0 \mathrm{a} \\
15.2 \pm 1.3 \mathrm{~b} \\
9 \pm 3.3 \mathrm{a} \\
210.1 \pm 14.8 \mathrm{~b}\end{array}$ & $\begin{array}{l}1.1 \pm 0.8 \mathrm{a} \\
1.5 \pm 0.1 \mathrm{ab} \\
0.2 \pm 0 \mathrm{a} \\
\text { n.d. } \\
78.4 \pm 3.6 \mathrm{a} \\
19 \pm 1.3 \mathrm{a} \\
10.1 \pm 1.1 \mathrm{a} \\
9.2 \pm 1.4 \mathrm{a} \\
9.9 \pm 1.3 \mathrm{a} \\
8.3 \pm 2.1 \mathrm{a} \\
137.7 \pm 5.1 \mathrm{a}\end{array}$ & $\begin{array}{l}1.9 \pm 0.1 \mathrm{a} \\
2.4 \pm 0 \mathrm{~b} \\
0.3 \pm 0 \mathrm{a} \\
\text { n.d. } \\
126.5 \pm 5.8 \mathrm{~b} \\
26.8 \pm 2 \mathrm{bc} \\
12 \pm 0.1 \mathrm{a} \\
12 \pm 1 \mathrm{a} \\
22.6 \pm 0.6 \mathrm{c} \\
9.3 \pm 0.4 \mathrm{a} \\
213.8 \pm 6.3 \mathrm{~b}\end{array}$ \\
\hline $\begin{array}{l}\text { 3,4-DHPEA } 1 \\
p \text {-HPEA } \\
\text { Vanilic acid } \\
p \text {-Cumaric acid } \\
\text { 3,4-DHPEA-EDA } \\
\text { p-HPEA-EDA } \\
\text { (+)-1-acetoxypinoresinol } \\
\text { (+)-pinoresinol } \\
\text { 3,4-DHPEA-EA } \\
\text { Ligstroside aglycone } \\
\text { Total phenols }\end{array}$ & $\begin{array}{l}\text { Moraiolo } \\
0.9 \pm 0.2 \mathrm{a} \\
1.2 \pm 0.4 \mathrm{a} \\
0.2 \pm 0 \mathrm{a} \\
0.3 \pm 0.1 \mathrm{a} \\
536.3 \pm 12 \mathrm{ac} \\
62.1 \pm 3.9 \mathrm{a} \\
29.8 \pm 0.6 \mathrm{a} \\
3.7 \pm 0.5 \mathrm{a} \\
71.2 \pm 4.6 \mathrm{a} \\
15.2 \pm 0.2 \mathrm{a} \\
720.8 \pm 14.2 \mathrm{ac}\end{array}$ & $\begin{array}{l}1 \pm 0.1 \mathrm{a} \\
1.3 \pm 0.1 \mathrm{a} \\
0.2 \pm 0 \mathrm{a} \\
0.3 \pm 0 \mathrm{a} \\
570.7 \pm 3.3 \mathrm{a} \\
60.7 \pm 3.9 \mathrm{a} \\
28.2 \pm 0.7 \mathrm{a} \\
7.3 \pm 5.8 \mathrm{a} \\
97.6 \pm 6.4 \mathrm{~b} \\
12.7 \pm 0.8 \mathrm{a} \\
780.2 \pm 12 \mathrm{a}\end{array}$ & $\begin{array}{l}1 \pm 0 \mathrm{a} \\
1.7 \pm 0.1 \mathrm{a} \\
0.3 \pm 0.1 \mathrm{a} \\
0.4 \pm 0 \mathrm{a} \\
417.8 \pm 13.6 \mathrm{~b} \\
56 \pm 1.1 \mathrm{a} \\
30.1 \pm 0.3 \mathrm{a} \\
15.1 \pm 0.9 \mathrm{a} \\
99.6 \pm 2.9 \mathrm{~b} \\
14.7 \pm 3.1 \mathrm{a} \\
636.7 \pm 14.6 \mathrm{~b}\end{array}$ & $\begin{array}{l}1.3 \pm 0.2 \mathrm{a} \\
1.6 \pm 0 \mathrm{a} \\
0.3 \pm 0 \mathrm{a} \\
0.4 \pm 0 \mathrm{a} \\
481.7 \pm 22.3 \mathrm{c} \\
57.5 \pm 2.1 \mathrm{a} \\
23.6 \pm 9.7 \mathrm{a} \\
13.5 \pm 0.3 \mathrm{a} \\
93.8 \pm 4.7 \mathrm{~b} \\
14.2 \pm 0.2 \mathrm{a} \\
687.7 \pm 25.3 \mathrm{bc}\end{array}$ \\
\hline $\begin{array}{l}\text { 3,4-DHPEA }{ }^{1} \\
p \text {-HPEA } \\
\text { Vanilic acid } \\
p \text {-Cumaric acid } \\
\text { 3,4-DHPEA-EDA } \\
\text { p-HPEA-EDA } \\
\text { (+)-1-acetoxypinoresinol } \\
\text { (+)-pinoresinol } \\
\text { 3,4-DHPEA-EA } \\
\text { Ligstroside aglycone } \\
\text { Total phenols }\end{array}$ & $\begin{array}{l}\text { Peranzana } \\
1.1 \pm 0.1 \mathrm{a} \\
3.2 \pm 0.4 \mathrm{a} \\
0.5 \pm 0.1 \mathrm{a} \\
0.3 \pm 0 \mathrm{a} \\
231.2 \pm 1.6 \mathrm{ac} \\
70.4 \pm 2.4 \mathrm{a} \\
10.8 \pm 2.5 \mathrm{a} \\
4.6 \pm 0.5 \mathrm{a} \\
53.5 \pm 0.5 \mathrm{ab} \\
12.1 \pm 0.7 \mathrm{a} \\
387.6 \pm 4 \mathrm{ac}\end{array}$ & $\begin{array}{l}0.9 \pm 0.4 \mathrm{a} \\
2.3 \pm 0.6 \mathrm{a} \\
0.3 \pm 0.2 \mathrm{a} \\
0.2 \pm 0.1 \mathrm{a} \\
279.6 \pm 6.1 \mathrm{~b} \\
71.8 \pm 3.9 \mathrm{a} \\
7.9 \pm 0.1 \mathrm{a} \\
4.3 \pm 0.3 \mathrm{a} \\
63.9 \pm 4.4 \mathrm{a} \\
13.9 \pm 1.8 \mathrm{a} \\
445 \pm 9.8 \mathrm{~b}\end{array}$ & $\begin{array}{l}0.9 \pm 0.1 \mathrm{a} \\
2 \pm 0.3 \mathrm{a} \\
0.2 \pm 0.1 \mathrm{a} \\
0 \pm 0 \mathrm{~b} \\
213.8 \pm 13.1 \mathrm{a} \\
60.2 \pm 1.1 \mathrm{~b} \\
5.6 \pm 0.4 \mathrm{a} \\
4.5 \pm 0.3 \mathrm{a} \\
50.2 \pm 4 \mathrm{~b} \\
12.4 \pm 1.1 \mathrm{a} \\
349.7 \pm 14.3 \mathrm{a}\end{array}$ & $\begin{array}{l}1 \pm 0.2 \mathrm{a} \\
3 \pm 0.5 \mathrm{a} \\
0.6 \pm 0.2 \mathrm{a} \\
0 \pm 0 \mathrm{~b} \\
240.5 \pm 7.3 \mathrm{a} \\
60.4 \pm 0.7 \mathrm{~b} \\
6.8 \pm 0.4 \mathrm{a} \\
4.2 \pm 0.2 \mathrm{a} \\
63.5 \pm 2.9 \mathrm{ab} \\
13 \pm 1.1 \mathrm{a} \\
393.1 \pm 8.5 \mathrm{c}\end{array}$ \\
\hline
\end{tabular}

${ }^{1}$ The data are the mean values of two independent extractions, \pm standard deviation. The values in each row with different letters (a-c) are significantly different from one another $(p<0.05)$. $\mathrm{C}=$ control (heat exchanger at $25^{\circ} \mathrm{C}$, malaxer at $25^{\circ} \mathrm{C}$ for $30 \mathrm{~min}$ ); $\mathrm{T} 1=$ test 1 (heat exchanger at $18{ }^{\circ} \mathrm{C}$, malaxer at $25^{\circ} \mathrm{C}$ for $30 \mathrm{~min}$ ); $\mathrm{T} 2=$ test 2 (heat exchanger at $18{ }^{\circ} \mathrm{C}$, malaxer at $18{ }^{\circ} \mathrm{C}$ for $30 \mathrm{~min}$ ); $\mathrm{T} 3=$ test 3 (heat exchanger at $18{ }^{\circ} \mathrm{C}$, malaxer at $18^{\circ} \mathrm{C}$ for $15 \mathrm{~min}+15 \mathrm{~min}$ at $25^{\circ} \mathrm{C}$ ).

The high enhancement of Canino was very interesting because the control content of phenolic compounds was very low and the use of a heat exchanger highly improved the sensory and health properties of the product.

The rapid reduction in the temperature of crushed olive paste (T1-VOO) also confirmed a significant influence of the cultivar on volatile compounds. The effects were strictly related to the different levels of the activity of the lipoxygenase (LOX) pathway enzymes that regulate the development of the VOO volatile fraction $[11,25]$.

For example, the T1-VOO of Canino showed a slight increase in the sum of saturated and unsaturated $C_{5}$ and $C_{6}$ aldehydes when compared to $\mathrm{C}-\mathrm{VOO}$, which was a difference that was not detected for the other two cultivars (Table 3). However, a slight reduction trend was common for the sum of the saturated and unsaturated $C_{5}$ and $C_{6}$ alcohols of $\mathrm{T} 1-\mathrm{VOO}$ in all the cultivars in comparison to that of the control oil extracted at $25^{\circ} \mathrm{C}$. The ester concentration, which was very low during the 2019 harvesting season, also existed in the cultivars, and was usually characterized by high content, such as in the case of 
Peranzana $[7,26]$, showing a reduction of content in Canino VOO and a slight increase in Peranzana VOO in Test 1 (Tables 3-5).

Table 3. Volatile composition $(\mu \mathrm{g} / \mathrm{kg})$ of Canino VOOs extracted at different temperatures.

\begin{tabular}{|c|c|c|c|c|}
\hline & C-VOO & T1-VOO & T2-VOO & T3-VOO \\
\hline \multicolumn{5}{|l|}{ Aldehydes ${ }^{1}$} \\
\hline Pentanal & $13 \pm 1 \mathrm{a}$ & $13 \pm 2 a$ & $15 \pm 1 \mathrm{a}$ & $10 \pm 1 \mathrm{a}$ \\
\hline (E)-2-Pentenal & $24 \pm 1 \mathrm{a}$ & $24 \pm 2 a$ & $35 \pm 3 b$ & $26 \pm 2 \mathrm{ab}$ \\
\hline Hexanal & $1072 \pm 79 a$ & $1075 \pm 78 \mathrm{a}$ & $1124 \pm 36 a$ & $961 \pm 37 a$ \\
\hline (E)-2-Hexenal & $23635 \pm 1162 a$ & $26416 \pm 1671 \mathrm{ac}$ & $35712 \pm 2257 b$ & $31580 \pm 987 \mathrm{bc}$ \\
\hline (E,E)-2,4-Hexadienal & $110 \pm 7 \mathrm{a}$ & $120 \pm 16 a$ & $120 \pm 5 a$ & $87 \pm 0 \mathrm{a}$ \\
\hline$\Sigma$ of aldehydes & $24855 \pm 1164 a$ & $27647 \pm 1673 \mathrm{ac}$ & $37006 \pm 2257 b$ & $32665 \pm 988 b c$ \\
\hline \multicolumn{5}{|l|}{ Alcohols } \\
\hline 1-Pentanol & $37 \pm 3 a$ & $22 \pm 2 b c$ & $26 \pm 2 b$ & $16 \pm 0 c$ \\
\hline 1-Penten-3-ol & $96 \pm 9 a$ & $103 \pm 11 b c$ & $139 \pm 8 b$ & $165 \pm 18 c$ \\
\hline (E)-2-Penten-1-ol & $11 \pm 1 a$ & $12 \pm 1 \mathrm{a}$ & $15 \pm 2 a$ & $39 \pm 3 b$ \\
\hline (Z)-2-Penten-1-ol & $91 \pm 5 a$ & $101 \pm 7 \mathrm{ab}$ & $134 \pm 12 b c$ & $153 \pm 8 c$ \\
\hline 1-Hexanol & $252 \pm 12 a$ & $180 \pm 18 b$ & $129 \pm 7 \mathrm{~b}$ & $184 \pm 19 b$ \\
\hline (E)-2-Hexen-1-ol & $652 \pm 28 a$ & $669 \pm 35 a$ & $367 \pm 24 b$ & $517 \pm 14 c$ \\
\hline (Z)-3-Esen-1-olo & $99 \pm 9 a$ & $102 \pm 5 a$ & $125 \pm 8 a$ & $182 \pm 8 b$ \\
\hline Benzyl alcohol & $69 \pm 8 a$ & $74 \pm 6 a$ & $77 \pm 1 \mathrm{a}$ & $71 \pm 1 \mathrm{a}$ \\
\hline Pheniletyl alcohol & $32 \pm 3 a$ & $32 \pm 3 a$ & $45 \pm 1 b$ & $39 \pm 3 a b$ \\
\hline$\Sigma$ of $C_{5}$ and $C_{6}$ alcohols & $1238 \pm 33 a$ & $1189 \pm 41 a$ & $935 \pm 30 b$ & $1256 \pm 32 a$ \\
\hline \multicolumn{5}{|l|}{ Esters } \\
\hline Hexyl acetate & $14 \pm 0 \mathrm{a}$ & $8 \pm 1 b$ & $5 \pm 1 b$ & $11 \pm 1 \mathrm{a}$ \\
\hline (Z)-3-Hexenyl acetate & $9 \pm 1 \mathrm{a}$ & $6 \pm 1 \mathrm{ab}$ & $7 \pm 0 \mathrm{ab}$ & $6 \pm 1 b$ \\
\hline$\Sigma$ of esters & $23 \pm 1 a$ & $14 \pm 1 \mathrm{bc}$ & $11 \pm 1 b$ & $17 \pm 1 c$ \\
\hline \multicolumn{5}{|l|}{ Ketones } \\
\hline 3-Pentanone + 2-Pentanone & $32 \pm 2 \mathrm{a}$ & $32 \pm 2 \mathrm{a}$ & $31 \pm 3 a$ & $31 \pm 1 \mathrm{a}$ \\
\hline 1-Penten-3-one & $195 \pm 14 \mathrm{a}$ & $181 \pm 8 \mathrm{a}$ & $295 \pm 15 b$ & $230 \pm 14 a$ \\
\hline 6-Methyl-5-hepten-2-one & $11 \pm 0 \mathrm{a}$ & $10 \pm 1 \mathrm{a}$ & $8 \pm 1 \mathrm{a}$ & $5 \pm 0 b$ \\
\hline$\Sigma$ of ketones & $238 \pm 14 a$ & $223 \pm 8 \mathrm{a}$ & $334 \pm 15 b$ & $266 \pm 14 a$ \\
\hline
\end{tabular}

${ }^{1}$ The data are the mean values of two independent extractions, \pm standard deviation. The values in each row with different letters (a-c) are significantly different from one another $(p<0.05)$. $\mathrm{C}=$ control (heat exchanger at $25^{\circ} \mathrm{C}$, malaxer at $25^{\circ} \mathrm{C}$ for 30 min); $\mathrm{T} 1=$ test 1 (heat exchanger at $18{ }^{\circ} \mathrm{C}$, malaxer at $25^{\circ} \mathrm{C}$ for $30 \mathrm{~min}$ ); $\mathrm{T} 2=$ test 2 (heat exchanger at $18{ }^{\circ} \mathrm{C}$, malaxer at $18{ }^{\circ} \mathrm{C}$ for 30 min); T3= test 3 (heat exchanger at $18{ }^{\circ} \mathrm{C}$, malaxer at $18^{\circ} \mathrm{C}$ for $15 \mathrm{~min}+15 \mathrm{~min}$ at $25^{\circ} \mathrm{C}$ ).

Table 4. Volatile composition $(\mu \mathrm{g} / \mathrm{kg})$ of Moraiolo VOOs extracted at different temperature.

\begin{tabular}{|c|c|c|c|c|}
\hline & C-VOO & T1-VOO & T2-VOO & T3-VOO \\
\hline \multicolumn{5}{|l|}{ Aldehydes ${ }^{1}$} \\
\hline Pentanal & $18 \pm 2 \mathrm{a}$ & $18 \pm 1 \mathrm{a}$ & $24 \pm 3 a$ & $22 \pm 0 \mathrm{a}$ \\
\hline (E)-2-Pentenal & $52 \pm 5 a$ & $44 \pm 4 a$ & $45 \pm 12 a$ & $49 \pm 2 a$ \\
\hline Hexanal & $830 \pm 1 \mathrm{a}$ & $865 \pm 53 a$ & $903 \pm 94 a$ & $657 \pm 68 \mathrm{a}$ \\
\hline (E)-2-Hexenal & $22141 \pm 800 \mathrm{a}$ & $22432 \pm 719 a$ & $24060 \pm 1344 a$ & $22091 \pm 1320 a$ \\
\hline (E,E)-2,4-Hexadienal & $86 \pm 5 \mathrm{ab}$ & $75 \pm 6 a$ & $99 \pm 4 b$ & $96 \pm 7 \mathrm{ab}$ \\
\hline$\Sigma$ of aldehydes & $23128 \pm 800 a$ & $23435 \pm 721 a$ & $25131 \pm 1348 a$ & $22915 \pm 1321 a$ \\
\hline \multicolumn{5}{|l|}{ Alcohols } \\
\hline 1-Pentanol & $23 \pm 3 a$ & $20 \pm 4 \mathrm{a}$ & $22 \pm 1 \mathrm{a}$ & $19 \pm 2 \mathrm{a}$ \\
\hline 1-Penten-3-ol & $294 \pm 21 a$ & $262 \pm 11 \mathrm{ab}$ & $216 \pm 7 b$ & $274 \pm 7 \mathrm{a}$ \\
\hline (E)-2-Penten-1-ol & $30 \pm 2 a$ & $24 \pm 5 \mathrm{a}$ & $19 \pm 2 a$ & $27 \pm 1 \mathrm{a}$ \\
\hline (Z)-2-Penten-1-ol & $307 \pm 21 a$ & $280 \pm 14 \mathrm{ab}$ & $223 \pm 15 b$ & $290 \pm 4 a$ \\
\hline 1-Hexanol & $450 \pm 7 a$ & $467 \pm 18 \mathrm{a}$ & $549 \pm 52 a$ & $450 \pm 35 a$ \\
\hline (E)-2-Hexen-1-ol & $679 \pm 8 a$ & $551 \pm 21 b c$ & $515 \pm 33 b$ & $665 \pm 41 \mathrm{ac}$ \\
\hline (Z)-3-Esen-1-olo & $449 \pm 42 \mathrm{a}$ & $426 \pm 41 \mathrm{a}$ & $515 \pm 12 \mathrm{a}$ & $460 \pm 39 a$ \\
\hline Benzyl alcohol & $67 \pm 2 a$ & $75 \pm 8 \mathrm{a}$ & $85 \pm 8 a$ & $77 \pm 3 a$ \\
\hline Pheniletyl alcohol & $78 \pm 1 \mathrm{a}$ & $93 \pm 7 a$ & $87 \pm 5 a$ & $75 \pm 4 a$ \\
\hline$\Sigma$ of $C_{5}$ and $C_{6}$ alcohols & $2231 \pm 53 a$ & $2031 \pm 52 \mathrm{a}$ & $2059 \pm 65 a$ & $2184 \pm 67 a$ \\
\hline
\end{tabular}


Table 4. Cont.

\begin{tabular}{|c|c|c|c|c|}
\hline & C-VOO & T1-VOO & T2-VOO & T3-VOO \\
\hline \multicolumn{5}{|l|}{ Esters } \\
\hline Hexyl acetate & $5 \pm 0 \mathrm{a}$ & $5 \pm 0 \mathrm{a}$ & $5 \pm 1 \mathrm{a}$ & $4 \pm 0 \mathrm{a}$ \\
\hline (Z)-3-Hexenyl acetate & $5 \pm 0 \mathrm{a}$ & $6 \pm 1 \mathrm{a}$ & $17 \pm 2 b$ & $8 \pm 1 \mathrm{a}$ \\
\hline$\Sigma$ of esters & $10 \pm 1 \mathrm{a}$ & $12 \pm 1 \mathrm{a}$ & $22 \pm 2 b$ & $13 \pm 1 \mathrm{a}$ \\
\hline \multicolumn{5}{|l|}{ Ketones } \\
\hline 3-Pentanone + 2-Pentanone & $121 \pm 4 \mathrm{a}$ & $103 \pm 13 a$ & $114 \pm 8 \mathrm{a}$ & $98 \pm 10 \mathrm{a}$ \\
\hline 1-Penten-3-one & $513 \pm 20 a$ & $410 \pm 7 b$ & $324 \pm 25 c$ & $453 \pm 16 a b$ \\
\hline 6-Methyl-5-hepten-2-one & $6 \pm 0 \mathrm{a}$ & $7 \pm 1 \mathrm{a}$ & $8 \pm 1 \mathrm{a}$ & $7 \pm 1 \mathrm{a}$ \\
\hline$\Sigma$ of ketones & $640 \pm 20 a$ & $520 \pm 14 b c$ & $445 \pm 27 b$ & $558 \pm 19$ ac \\
\hline
\end{tabular}

${ }^{1}$ The data are the mean values of two independent extractions, \pm standard deviation. The values in each row with different letters (a-c) are significantly different from one another $(p<0.05) . \mathrm{C}=$ control (heat exchanger at $25^{\circ} \mathrm{C}$, malaxer at $25^{\circ} \mathrm{C}$ for $30 \mathrm{~min}$ ); $\mathrm{T} 1=$ test 1 (heat exchanger at $18{ }^{\circ} \mathrm{C}$, malaxer at $25^{\circ} \mathrm{C}$ for $30 \mathrm{~min}$ ); $\mathrm{T} 2=$ test 2 (heat exchanger at $18{ }^{\circ} \mathrm{C}$, malaxer at $18{ }^{\circ} \mathrm{C}$ for $30 \mathrm{~min}$ ); $\mathrm{T} 3=$ test 3 (heat exchanger at $18{ }^{\circ} \mathrm{C}$, malaxer at $18^{\circ} \mathrm{C}$ for $15 \mathrm{~min}+15 \mathrm{~min}$ at $\left.25^{\circ} \mathrm{C}\right)$.

Table 5. Volatile composition $(\mu \mathrm{g} / \mathrm{kg})$ of Peranzana VOOs extracted at different temperatures.

\begin{tabular}{|c|c|c|c|c|}
\hline & C-VOO & T1-VOO & T2-VOO & T3-VOO \\
\hline \multicolumn{5}{|l|}{ Aldehydes ${ }^{1}$} \\
\hline Pentanal & $17 \pm 0 \mathrm{a}$ & $10 \pm 1 b$ & $15 \pm 1 \mathrm{a}$ & $12 \pm 2 \mathrm{a}$ \\
\hline (E)-2-Pentenal & $68 \pm 3 a$ & $42 \pm 18 a$ & $55 \pm 26 a$ & $38 \pm 1 \mathrm{a}$ \\
\hline Hexanal & $925 \pm 59 a$ & $1321 \pm 12 \mathrm{a}$ & $1388 \pm 481 \mathrm{a}$ & $1150 \pm 144 a$ \\
\hline (E)-2-Hexenal & $17306 \pm 1308 a$ & $15181 \pm 1771 a$ & $18043 \pm 476 a$ & $17045 \pm 935 a$ \\
\hline (E,E)-2,4-Hexadienal & $133 \pm 13 a$ & $139 \pm 30 a$ & $143 \pm 61 a$ & $123 \pm 33 a$ \\
\hline$\Sigma$ of aldehydes & $18450 \pm 1309 a$ & $16693 \pm 1772 a$ & $19644 \pm 680 a$ & $18368 \pm 947 a$ \\
\hline \multicolumn{5}{|l|}{ Alcohols } \\
\hline 1-Pentanol & $18 \pm 3 a$ & $20 \pm 1 \mathrm{a}$ & $19 \pm 0 \mathrm{a}$ & $17 \pm 2 \mathrm{a}$ \\
\hline 1-Penten-3-ol & $340 \pm 11 a$ & $325 \pm 20 a$ & $283 \pm 168 a$ & $166 \pm 3 a$ \\
\hline (E)-2-Penten-1-ol & $28 \pm 0 \mathrm{a}$ & $16 \pm 9 a$ & $21 \pm 12 \mathrm{a}$ & $13 \pm 0 \mathrm{a}$ \\
\hline (Z)-2-Penten-1-ol & $360 \pm 6 a$ & $252 \pm 51 \mathrm{ab}$ & $178 \pm 24 b$ & $150 \pm 15 b$ \\
\hline 1-Hexanol & $147 \pm 7 \mathrm{a}$ & $103 \pm 8 \mathrm{a}$ & $96 \pm 37 a$ & $64 \pm 8 a$ \\
\hline (E)-2-Hexen-1-ol & $143 \pm 22 a$ & $107 \pm 7 \mathrm{ab}$ & $86 \pm 8 b$ & $74 \pm 9 b$ \\
\hline (Z)-3-Esen-1-olo & $282 \pm 9 a$ & $161 \pm 21 \mathrm{a}$ & $177 \pm 112 \mathrm{a}$ & $91 \pm 9 a$ \\
\hline Benzyl alcohol & $71 \pm 3 a$ & $70 \pm 7 a$ & $88 \pm 31 a$ & $63 \pm 4 a$ \\
\hline Pheniletyl alcohol & $61 \pm 8 \mathrm{a}$ & $38 \pm 3 a$ & $45 \pm 23 a$ & $27 \pm 2 \mathrm{a}$ \\
\hline$\Sigma$ of $C_{5}$ and $C_{6}$ alcohols & $1318 \pm 27 a$ & $985 \pm 60 \mathrm{ab}$ & $860 \pm 207 b$ & $575 \pm 22 b$ \\
\hline \multicolumn{5}{|l|}{ Esters } \\
\hline Hexyl acetate & $8 \pm 4 a$ & $52 \pm 19 \mathrm{ab}$ & $28 \pm 16 \mathrm{ab}$ & $65 \pm 12 b$ \\
\hline (Z)-3-Hexenyl acetate & $124 \pm 6 a$ & $148 \pm 21 \mathrm{a}$ & $168 \pm 9 a$ & $159 \pm 10 a$ \\
\hline$\sum$ of esters & $132 \pm 7 a$ & $201 \pm 28 \mathrm{ab}$ & $196 \pm 19 \mathrm{ab}$ & $224 \pm 16 b$ \\
\hline \multicolumn{5}{|l|}{ Ketones } \\
\hline 3-Pentanone + 2-Pentanone & $26 \pm 1 \mathrm{a}$ & $13 \pm 7 \mathrm{a}$ & $25 \pm 4 \mathrm{a}$ & $11 \pm 2 \mathrm{a}$ \\
\hline 1-Penten-3-one & $662 \pm 29 a$ & $367 \pm 169 \mathrm{ab}$ & $270 \pm 25 b$ & $680 \pm 40 a$ \\
\hline 6-Methyl-5-hepten-2-one & $8 \pm 0 \mathrm{a}$ & $8 \pm 1 \mathrm{a}$ & $8 \pm 0 \mathrm{a}$ & $9 \pm 0 \mathrm{a}$ \\
\hline$\Sigma$ of ketones & $696 \pm 29 a$ & $389 \pm 169 \mathrm{ab}$ & $303 \pm 25 b$ & $699 \pm 40 a$ \\
\hline
\end{tabular}

${ }^{1}$ The data are the mean values of two independent extractions, \pm standard deviation. The values in each row with different letters (a-c) are significantly different from one another $(p<0.05) . \mathrm{C}=$ control (heat exchanger at $25^{\circ} \mathrm{C}$, malaxer at $25^{\circ} \mathrm{C}$ for $30 \mathrm{~min}$ ); $\mathrm{T} 1=$ test 1 (heat exchanger at $18{ }^{\circ} \mathrm{C}$, malaxer at $25^{\circ} \mathrm{C}$ for $30 \mathrm{~min}$ ); $\mathrm{T} 2=$ test 2 (heat exchanger at $18{ }^{\circ} \mathrm{C}$, malaxer at $18{ }^{\circ} \mathrm{C}$ for $30 \mathrm{~min}$ ); $\mathrm{T} 3=$ test 3 (heat exchanger at $18{ }^{\circ} \mathrm{C}$, malaxer at $18^{\circ} \mathrm{C}$ for $15 \mathrm{~min}+15 \mathrm{~min}$ at $25^{\circ} \mathrm{C}$ ).

\subsection{Tests 2 and 3-Use of Low Temperature during the Malaxation Phase}

The other two experimental trials (Tests 2 and 3) also prolonged the use of low temperature during the malaxation phase to evaluate the eventual positive effects on the formation of volatile compounds and on the hydrophilic phenol content of VOOs. The data obtained from Test 2 and Test 3 showed a differentiated impact on VOO quality parameters due to the different cultivars, probably as a consequence of the different activities of endogenous enzymes that regulate volatile and phenolic compounds. In fact, the LOX pathway is characterized by different levels of enzymatic activity, which are influenced by 
the genetic origin of the fruits, and this explains the variable development of the volatile aldehydes, alcohols and ester compounds that determine the large aromatic variability of VOO, as explained by other authors [25,27].

The different LOX activities are influenced not only by agronomic practices, the growing area, and the ripening index but also by the temperature of the extraction process $[4,8,11,28]$. With the use of low temperature during the entire cycle of malaxation (T2-VOO) and by extending the time of optimum temperature of LOX activity [29], a further significant increase in (E)-2-exenal in VOO of the Canino cultivar was observed. The sum of the aldehydes mainly showed an enhancement of $48.9 \%$ and $33.9 \%$ of T2-VOO compared with the enhancements seen in C-VOO and T1-VOO (Table 3). On the contrary, alcohols showed an opposite trend, with a significant reduction in the sum of $\mathrm{C}_{5}$ - and $\mathrm{C}_{6}$-saturated and unsaturated alcohols of $24.5 \%$ compared to the C-VOO.

Although significant differences have been pointed out among the different tests, the concentration of esters of Canino VOO was probably too low to recognize a variability in the sensory properties of the product $[30,31]$. When reducing the period of low temperature during the malaxation phase to $15 \mathrm{~min}$ (T3-VOO), the volatile fraction of the Canino cultivar showed a decrease in the sum of aldehydes compared with T2-VOO but a higher value compared with $\mathrm{C}-\mathrm{VOO}$ and T1-VOO (Table 3). The results of the Canino cultivar suggest a close connection between the increase in aldehydes and the use of low temperature during the crushing and malaxation phases, which could have significant enhancements depending on the time spent by olive paste at $18^{\circ} \mathrm{C}$. The prolongation of low temperature seems to improve the activity of specific hydroperoxide lyases that generate the main $\mathrm{C}_{6}$ aldehydes. In contrast, the application of low temperature during the kneading of olive paste showed a significant impact on the volatile sensory note of Canino VOO with an increase in green and leaf-like aroma due to (E)-2-exenal [30] and a reduction in fruity and green sweet sensory notes due to the main alcohols [29,32]. Similar data and the high influence of cultivars on different synthesis of $C_{5}$ and $C_{6}$ volatile compounds was also recently proposed by other authors $[6,33,34]$.

On the other hand, the use of low temperature during the malaxation phase reduced the phenolic content of Canino T2-VOO by 34\% compared to T1-VOO, whereas the value rose again in Test 3 when the low temperature was maintained for only half of the kneading process. The data highlighted that the inhibitory effect of low temperature on PPO and POD activity [5] was minor compared to the effect of the solubilization process of phenolic compounds in the oily phase induced by the application of higher temperature $\left(25^{\circ} \mathrm{C}\right)$ during the malaxation step (Table 2). Qualitatively, the main differences were observed for oleuropein and ligstroside derivatives such as 3,4-DHPEA-EDA, p-HPEA-EDA and 3,4-DHPEA-EA. The VOO phenolic concentrations of the other two cultivars (Moraiolo and Peranzana) showed the same trend as Canino VOOs (Table 2). The prolonged use of low temperature for the entire cycle of the malaxation step (Test 2) confirmed that the reduction of partition processes in the phenolic fraction between aqueous and oily phases of olive paste occurred with decreases of $18.3 \%$ and $21.4 \%$ for T2-VOO hydrophilic phenol content compared to T1-VOOs for Moraiolo and Peranzana. However, the reduction trend was more limited if the results of T2-VOOs were compared with the C-VOOs, and there were differences that were not statistically significant for Canino and Peranzana cultivars. These results were significant in confirming the high impact of the rapid thermal conditioning of crushed olive paste (Test 1), which highlighted the importance of the use of a heat exchanger in oil mechanical extraction plants to improve the phenolic fraction of VOOs and, as a consequence, the health and sensory properties of the product $[8,17,20-22]$.

Another interesting result, which was also confirmed by the Moraiolo and Peranzana cultivars, concerned the evolution of VOO phenolic compounds during Test 3, in which increasing the temperature at $25^{\circ} \mathrm{C}$ for the last $15 \mathrm{~min}$ of malaxation time was enough again to improve the levels of solubilization of the phenolic fraction into the oily phase of olive paste. The T3-VOOs enhanced their concentration of $8 \%$ and $12.4 \%$ compared with 
the trials conducted at $18{ }^{\circ} \mathrm{C}$ for the entire malaxation time for Moraiolo and Peranzana cultivars (Table 2).

In contrast, the genetic origin of the fruits had a major influence on the evolution of VOO volatile compounds both in quantitative and qualitative terms. In fact, the impact of different temperatures used after the crushing phase or during malaxation highlighted different evolution trends for the other two cultivars compared with the concentrations of the volatile fraction of Canino VOOs. The sum of aldehydes in Test 2 slightly increased, and above all in the Moraiolo VOO, even if the data were not statistically significant. Concerning the sum of alcohols and esters, the results showed a reduction and an increase in their concentration, respectively, compared to the C-VOO of Peranzana. The alcohols of Moraiolo showed the same evolution trend even if the data were not statistically significant, whereas the ester content, as analyzed for Canino VOOs, was too low to recognize the effect of a significant increase (Tables 4 and 5). During the technological tests on the optimization of the oil mechanical extraction process, the use of low temperature showed a constant impact on the VOO phenolic content, whereas the evolution of volatile compounds seemed to be more complex with different responses in function for the different olives processed. As reported by other studies on VOO technology, the quantitative and qualitative volatile fraction is first influenced by the genetic origin of the fruits, which are characterized by different behaviors when different technological parameters are modified [4,8,20,24-27,33-38].

\section{Conclusions}

The first result of this study confirmed the positive role of rapid thermal conditioning on the olive paste of different cultivars after the crushing phase, which was induced by the reduction of temperature using a heat exchanger. The impact was mainly related to the improvement of the hydrophilic phenol content of VOOs in all the cultivars processed with different quantitative increases. In contrast, the low temperature, also applied during the malaxation phase, determined an overall reduction in the phenolic fraction. The data highlighted how the solubilization phenomenon of phenolic compounds into the oily phase, promoted at $25{ }^{\circ} \mathrm{C}$ of extraction temperature, played a higher role in the determination of the final VOO phenolic concentration than the impact of a probable reduction in the enzymatic oxidation induced by the use of lower temperature during the kneading of olive paste.

The impact on the volatile fraction, which influenced the VOO flavor, was strongly conditioned by the genetic origin of the olives, which determined different responses to the application of low temperature during the malaxation phase. The VOO of the Canino cultivar was the only cultivar that showed a significant evolution of the volatile fraction, with a higher content of the sum of aldehydes and a reduction of the sum of alcohols that contributed to an enhancement of the "green" sensory note of VOOs. The use of low temperature for half of the malaxation phase could represent, for the Canino cultivar, a middle course to optimize the reduction effect of phenolic compounds and the increase of aldehydes, whereas for the other cultivars, which did not show significant improvement in volatile concentration, application of $18{ }^{\circ} \mathrm{C}$ temperature during the kneading step would not be recommended.

Knowledge of new technologies and the settling of different technological parameters, such as temperature and time of treatment, should be implemented to optimize the mechanical extraction process as a function of the different cultivars processed, with the opportunity of obtaining VOO of higher quality standards. The high variability was also apparent in this study and mainly related to the volatile content evolution. This variability suggests the importance of having a range of solutions to extract from each different cultivar, different VOOs characterized by different levels of volatile and phenolic compounds that can characterize the sensory and health properties of the product and intercept with different groups of consumers. 
Author Contributions: Conceptualization, S.E., A.T., D.N. and G.V.; methodology, D.N., R.S. and G.V.; formal analysis, D.N. and R.S.; investigation, R.T., M.P., M.S. and G.V.; data curation, R.S., D.N. and G.V. writing-original draft preparation, G.V.; writing-review and editing, G.V.; visualization S.E., A.T. and G.V. supervision, M.S.; project administration, R.T., M.P. and M.S.; funding acquisition, M.S. All authors have read and agreed to the published version of the manuscript.

Funding: This research was funded by Ministero delle Politiche Agricole Alimentari Forestali (Mipaaf)_research project OLIVENEWTECH-ID 04 (D.M. n.18200) and INFOLIVA (D.M. n.12479).

Acknowledgments: We thank Roberto Santibacci (University of Perugia) for technical assistance of olive oil mechanical extraction process.

Conflicts of Interest: The authors declare no conflict of interest.

\section{References}

1. Clodoveo, M.L.; Hbaieb, R.H.; Kotti, F.; Mugnozza, G.S.; Gargouri, M. Mechanical Strategies to Increase Nutritional and Sensory Quality of Virgin Olive Oil by Modulating the Endogenous Enzyme Activities. Compr. Rev. Food Sci. Food Saf. 2014, 13, 135-154. [CrossRef]

2. Luaces, P.; Sanz, C.; Pérez, A.G. Thermal Stability of Lipoxygenase and Hydroperoxide Lyase from Olive Fruit and Repercussion on Olive Oil Aroma Biosynthesis. J. Agric. Food Chem. 2007, 55, 6309-6313. [CrossRef] [PubMed]

3. Marx, Í.M.; Rodrigues, N.; Veloso, A.C.; Casal, S.; Pereira, J.A.; Peres, A.M. Effect of malaxation temperature on the physicochemical and sensory quality of cv. Cobrançosa olive oil and its evaluation using an electronic tongue. LWT 2021, 137, 110426. [CrossRef]

4. Selvaggini, R.; Esposto, S.; Taticchi, A.; Urbani, S.; Veneziani, G.; Di Maio, I.; Sordini, B.; Servili, M. Optimization of the temperature and oxygen concentration conditions in the malaxation during the oil mechanical extraction process of four Italian olive cultivars. J. Agric. Food Chem. 2014, 62, 3813-3822. [CrossRef] [PubMed]

5. Taticchi, A.; Esposto, S.; Veneziani, G.; Urbani, S.; Selvaggini, R.; Servili, M. The influence of the malaxation temperature on the activity of polyphenoloxidase and peroxidase and on the phenolic composition of virgin olive oil. Food Chem. 2013, 136, 975-983. [CrossRef]

6. Vidal, A.M.; Alcala, S.; De Torres, A.; Moya, M.; Espinola, J.M.; Espinola, F. Fresh and aromatic virgin olive oil obtained from Arbequina, Koroneiki, and Arbosana cultivars. Molecules 2019, 24, 3587. [CrossRef] [PubMed]

7. Veneziani, G.; Esposto, S.; Taticchi, A.; Selvaggini, R.; Urbani, S.; Di Maio, I.; Sordini, B.; Servili, M. Flash Thermal Conditioning of Olive Pastes during the Oil Mechanical Extraction Process: Cultivar Impact on the Phenolic and Volatile Composition of Virgin Olive Oil. J. Agric. Food Chem. 2015, 63, 6066-6074. [CrossRef] [PubMed]

8. Veneziani, G.; Esposto, S.; Taticchi, A.; Urbani, S.; Selvaggini, R.; Sordini, B.; Servili, M. Characterization of phenolic and volatile composition of extra virgin olive oil extracted from six Italian cultivars using a cooling treatment of olive paste. LWT 2018, 87, 523-528. [CrossRef]

9. Miho, H.; Moral, J.; López-González, M.A.; Díez, C.M.; Priego-Capote, F. The phenolic profile of virgin olive oil is influenced by malaxation conditions and determines the oxidative stability. Food Chem. 2020, 314, 126183. [CrossRef]

10. Angerosa, F.; Mostallino, R.; Basti, C.; Vito, R. Influence of malaxation temperature and time on the quality of virgin olive oils. Food Chem. 2001, 72, 19-28. [CrossRef]

11. Sánchez-Ortiz, A.; Romero-Segura, C.; Sanz, C.; Pérez, A.G. Synthesis of Volatile Compounds of Virgin Olive Oil Is Limited by the Lipoxygenase Activity Load during the Oil Extraction Process. J. Agric. Food Chem. 2012, 60, 812-822. [CrossRef]

12. Ridolfi, M.; Terenziani, S.; Patumi, M.; Fontanazza, G. Characterization of the Lipoxygenases in Some Olive Cultivars and Determination of Their Role in Volatile Compounds Formation. J. Agric. Food Chem. 2002, 50, 835-839. [CrossRef]

13. Salas, J.J.; Sanchez, J. The decrease of virgin olive oil flavor produced by high malaxation temperature is due to inactivation of hydroperoxide lyase. J. Agric. Food Chem. 1999, 47, 809-812. [CrossRef]

14. Luaces, P.; Pérez, A.G.; Sanz, C. Effect of the blanching process and olive fruit temperature at milling on the biosynthesis of olive oil aroma. Eur. Food Res. Technol. 2006, 224, 11-17. [CrossRef]

15. Padilla, M.N.; Martínez-Rivas, J.M.; Pérez, A.G.; Sanz, C. Thermal Inactivation Kinetics of Recombinant Proteins of the Lipoxygenase Pathway Related to the Synthesis of Virgin Olive Oil Volatile Compounds. J. Agric. Food Chem. 2012, 60, 6477-6482. [CrossRef] [PubMed]

16. Dourou, A.-M.; Brizzolara, S.; Meoni, G.; Tenori, L.; Famiani, F.; Luchinat, C.; Tonutti, P. The inner temperature of the olives (cv. Leccino) before processing affects the volatile profile and the composition of the oil. Food Res. Int. 2020, 129, 108861. [CrossRef]

17. Veneziani, G.; Esposto, S.; Taticchi, A.; Urbani, S.; Selvaggini, R.; Di Maio, I.; Sordini, B.; Servili, M. Cooling treatment of olive paste during the oil processing: Impact on the yield and extra virgin olive oil quality. Food Chem. 2017, 221, 107-113. [CrossRef] [PubMed]

18. Angerosa, F.; Servili, M.; Selvaggini, R.; Taticchi, A.; Esposto, S.; Montedoro, G.F. Volatile compounds in virgin olive oil: Occurrence and their relationship with the quality. J. Chromatogr. A 2004, 1054, 17-31. [CrossRef] 
19. Caporaso, N. Virgin Olive Oils: Environmental Conditions, Agronomical Factors and Processing Technology Affecting the Chemistry of Flavor Profile. J. Food Chem. Nanotechnol. 2016, 2, 21-31. [CrossRef]

20. Caponio, F.; Leone, A.; Squeo, G.; Tamborrino, A.; Summo, C. Innovative technologies in virgin olive oil extraction process: Influence on volatile compounds and organoleptic characteristics. J. Sci. Food Agric. 2019, 99, 5594-5600. [CrossRef]

21. Cecchi, L.; Bellumori, M.; Corbo, F.; Milani, G.; Clodoveo, M.L.; Mulinacci, N. Implementation of the sono-heat-exchanger in the extra virgin olive oil extraction process: End-user validation and analytical evaluation. Molecules 2019, 24, 2379-2391. [CrossRef]

22. Leone, A.; Esposto, S.; Tamborrino, A.; Romaniello, R.; Taticchi, A.; Urbani, S.; Servili, M. Using a tubular heat exchanger to improve the conditioning process of the olive paste: Evaluation of yield and olive oil quality. Eur. J. Lipid Sci. Technol. 2016, 118, 308-317. [CrossRef]

23. OJEC-Commission Delegated Regulation (EU). 2015/1830 of 8 July 2015 amending Regulation (EEC) No $2568 / 91$ on the characteristics of olive oil and olive-residue oil and on the relevant methods of analysis. Off. J. Eur. Union. 2015, 266, 9-13.

24. Taticchi, A.; Esposto, S.; Veneziani, G.; Minnocci, A.; Urbani, S.; Selvaggini, R.; Sordini, B.; Daidone, L.; Sebastiani, L.; Servili, M. High vacuum-assisted extraction affects virgin olive oil quality: Impact on phenolic and volatile compounds. Food Chem. 2021, 342, 128369. [CrossRef] [PubMed]

25. Aprea, E.; Gasperi, F.; Betta, E.; Sani, G.; Cantini, C. Variability in volatile compounds from lipoxygenase pathway in extra virgin olive oils from Tuscan olive germoplasm by quantitative SPME/GC-MS. J. Mass Spec. 2018, 53, 824-832. [CrossRef] [PubMed]

26. Esposto, S.; Veneziani, G.; Taticchi, A.; Selvaggini, R.; Urbani, S.; Di Maio, I.; Sordini, B.; Servili, M. Flash thermal conditioning of olive pastes during the olive oil mechanical extraction process: Impact on the structural modifications of pastes and oil quality. $J$. Agric. Food Chem. 2013, 61, 4953-4960. [CrossRef] [PubMed]

27. García-Vico, L.; Belaj, A.; Sánchez-Ortiz, A.; Martínez-Rivas, J.M.; Pérez, A.; Sanz, C. Volatile compound profiling by HSSPME/GC-MS-FID of a core olive cultivar collection as a tool for aroma improvement of virgin olive oil. Molecules 2017, 22, 141. [CrossRef]

28. Hbaieb, R.H.; Kotti, F.; Vichi, S.; Gargouri, M. Evolution of endogenous enzyme activities and virgin olive oil characteristics during Chétoui and Chemlali olive ripening. Eur. J. Lipid Sci. Technol. 2017, 119, 1600150. [CrossRef]

29. Kalua, C.M.; Allen, M.S.; Bedgood, D.R.; Bishop, A.G.; Prenzler, P.D.; Robards, K. Olive oil volatile compounds, flavour development and quality: A critical review. Food Chem. 2007, 100, 273-286. [CrossRef]

30. Yan, J.; Alewijn, M.; Van Ruth, S.M. From Extra Virgin Olive Oil to Refined Products: Intensity and Balance Shifts of the Volatile Compounds versus Odor. Molecules 2020, 25, 2469. [CrossRef]

31. Pérez, A.G.; de la Rosa, R.; Pascual, M.; Sánchez-Ortiz, A.; Romero-Segura, C.; León, L.; Sanz, C. Assessment of volatile compound profiles and the deduced sensory significance of virgin olive oils from the progeny of Picual $\times$ Arbequina cultivars. J. Chromatogr. A 2016, 1428, 305-315. [CrossRef] [PubMed]

32. Luna, G.; Morales, M.; Aparicio, R. Characterisation of 39 varietal virgin olive oils by their volatile compositions. Food Chem. 2006, 98, 243-252. [CrossRef]

33. Tomé-Rodríguez, S.; Ledesma-Escobar, C.A.; Penco-Valenzuela, J.M.; Priego-Capote, F. Cultivar influence on the volatile components of olive oil formed in the lipoxygenase pathway. LWT 2021, 147, 111485. [CrossRef]

34. Cherfaoui, M.; Cecchi, T.; Keciri, S.; Boudriche, L. Volatile and Sensory Profiles of Algerian Extra-Virgin Olive Oil from Souidi and Zeletni Cultivars. Chem. Biodivers. 2019, 16, e1900297. [CrossRef]

35. Kalogianni, E.P.; Georgiou, D.; Hasanov, J.H. Olive oil processing: Current knowledge, literature gaps, and future perspectives. J. Am. Oil Chem. Soc. 2019, 96, 481-507. [CrossRef]

36. Pérez, M.; López-Yerena, A.; Lozano-Castellón, J.; Olmo-Cunillera, A.; Lamuela-Raventós, R.M.; Martin-Belloso, O.; VallverdúQueralt, A. Impact of Novel Technologies on Virgin Olive Oil Processing, Consumer Acceptance, and the Valorization of Olive Mill Wastes. Antioxidants 2021, 10, 417. [CrossRef]

37. Taticchi, A.; Selvaggini, R.; Esposto, S.; Sordini, B.; Veneziani, G.; Servili, M. Physicochemical characterization of virgin olive oil obtained using an ultrasound-assisted extraction at an industrial scale: Influence of olive maturity index and malaxation time. Food Chem. 2019, 289, 7-15. [CrossRef]

38. Veneziani, G.; Esposto, S.; Taticchi, A.; Selvaggini, R.; Sordini, B.; Lorefice, A.; Daidone, L.; Pagano, M.; Tomasone, R.; Servili, M. Extra-Virgin Olive Oil Extracted Using Pulsed Electric Field Technology: Cultivar Impact on Oil Yield and Quality. Front. Nutr. 2019, 6, 134. [CrossRef] [PubMed] 